\title{
Overnight urinary 11-hydroxycorticosteroid estimations in diagnosis of Cushing's syndrome
}

\section{Summary}

Overnight specimens of urine were collected from control, obese, and hirsute women and from 11 women with Cushing's syndrome. Urinary 11-hydroxycorticosteroid levels in the group with Cushing's syndrome were significantly higher than in the other three groups. This simple test has proved invaluable when screening for Cushing's syndrome in a busy outpatient clinic.

\section{Introduction}

Some years ago we described a simple screening test for Cushing's syndrome based on the estimation of 11-hydroxycorticosteroids in a 24-hour specimen of urine. ${ }^{1}$ We found this test invaluable in identifying those patients who required the more extensive investigations needed to confirm the diagnosis and determine the aetiology. Nevertheless, the accurate collection of 24-hour urine specimens by outpatients is not without its difficulties. Since the circadian rhythm of steroid excretion is abnormal in Cushing's syndrome, ${ }^{2}{ }^{3}$ we thought that an overnight collection of urine might be sufficient to identify those patients who were secreting excessive amounts of cortisol.

We report our results in normal controls, obese and hirsute patients, and patients with Cushing's syndrome. Because the normal ranges of urinary 11-hydroxycorticosteroids are different in men and women we confined our observations to women. In any case Cushing's syndrome is far commoner in women than in men.

\section{Subjects and methods}

We studied four groups of women. The first group consisted of 29 healthy women, outpatients, and convalescent inpatients. The inpatients were not acutely ill and were under no obvious stress at the time of the urine collection. Patients with endocrine, liver, or renal diseases were not included. The second group consisted of 49 women who were obviously obese but who had no other manifestation of Cushing's syndrome. The third group consisted of 38 women who showed considerable hirsutism. Eleven of these had polycystic ovaries. Twenty were obese as well as hirsute. In some of these obese and hirsute patients, including all those with an overnight 11-hydroxycorticosteroid excretion (expressed in cortisol equivalents) of more than $221 \mathrm{nmol}(80 \mu \mathrm{g})$, Cushing's syndrome was excluded using the investigations described in another paper. ${ }^{4}$ The fourth group consisted of 11 women with Cushing's syndrome due to bilateral adrenal hyperplasia. In all cases the normal circadian variation of the plasma 11-hydroxycorticosteroid levels was absent or impaired, and the plasma and urinary 11-hydroxycorticosteroid levels were raised and failed to fall normally during dexamethasone administration. The diagnosis was confirmed by inspecting the adrenal glands at operation in nine cases. A total of 22 overnight urine specimens were collected from these 11 patients.

Postgraduate Medical Institute, University of Exeter, Exeter EX2 5DW

D MATTINGLY, MB, FRCP, director

CHRISTINE TYLER, BSC, PHD, research fellow
Urine collections-In seven controls and three of the patients with Cushing's syndrome urine was collected over 24 hours in three eighthour periods: from 0600 to 1400 , from 1400 to 2200 , and from 2200 to 0600 . In the remaining subjects overnight urine collections were made from the time they went to bed to when they got up in the morning. They were given written instructions telling them to empty the bladder normally last thing before going to bed and to collect all urine passed during the night and on rising. The urine was collected through large polyethylene funnels into wide-necked one-litre polyethylene bottles that had been prewashed with the detergent Decon-75. Specimens were brought to the laboratory as soon as possible after being collected. Any samples arriving after noon were discarded.

11-hydroxycorticosteroids were determined with the fluorimetric method of Mattingly et al, ${ }^{5}$ using $2-\mathrm{ml}$ samples of urine.

\section{Results}

Our results confirmed the existence of a strong circadian rhythm in the excretion of urinary 11-hydroxycorticosteroids in normal people, with the smallest proportion of the 24-hour excretion occurring during the night. In the three patients with Cushing's syndrome, however, no similar pattern was discerned, and the overnight excretion of urinary 11-hydroxycorticosteroids was much higher than that seen in the control group (see table).

Circadian rhythms of urinary 11-hydroxycorticosteroids in three patients with Cushing's syndrome and seven control subjects (mean $\pm S D$ values)

\begin{tabular}{c|c|c|c}
\hline \multirow{2}{*}{ Case No } & \multicolumn{2}{|c}{ 11-hydroxycorticosteroids $(\mathrm{nmol} / 8 \mathrm{~h})$} \\
\cline { 2 - 3 } & $0600-1400$ & $1400-2200$ & $2200-0600$ \\
\hline 1 & 1391 & 2608 & 1214 \\
2 & 370 & 626 & 392 \\
3 & 483 & 317 & 580 \\
\hline Controls & $295 \pm 105$ & $171 \pm 52$ & $116 \pm 50$ \\
\hline
\end{tabular}

Conversion: SI to traditional units-11-hydroxycorticosteroids: $1 \mathrm{nmol} / 8 \mathrm{~h} \approx 0.36$ $\mu \mathrm{g} / 8 \mathrm{~h}$.

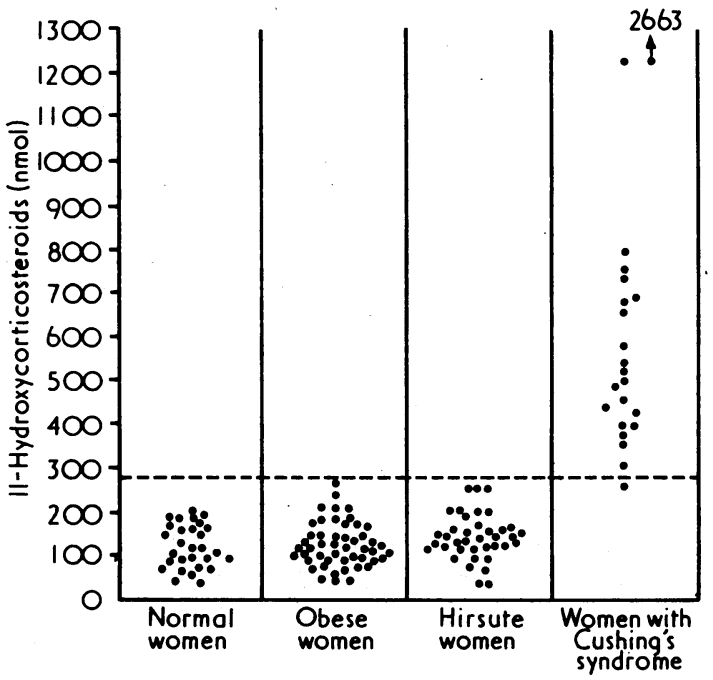

Overnight urine values in all subjects. Twenty-two specimens were collected from the 11 women with Cushing's syndrome. Conversion: SI to traditional units-11-hydroxycorticosteroids: $1 \mathrm{nmol} \approx 0.36 \mu \mathrm{g}$ 
The results on the overnight urine specimens in all the subjects are shown in the figure. The mean overnight 11-hydroxycorticosteroid levels and the standard deviations in the control, obese, and hirsute groups were similar, being $119 \pm 47 \mathrm{nmol}(43 \pm 17 \mathrm{~kg}), 121 \pm 50 \mathrm{nmol}$ $(44 \pm 18 \mu \mathrm{g})$, and $138 \pm 55 \mathrm{nmol}(50 \pm 20 \mathrm{~kg})$ respectively. The highest value of $271 \mathrm{nmol}(98 \mathrm{~kg})$ occurred in an obese patient. By contrast, only one of the patients with Cushing's syndrome had a urinary 11-hydroxycorticosteroid excretion of less than $276 \mathrm{nmol}(100 \mu \mathrm{g})$ during the night. On the night in question her midnight plasma 11-hydroxycorticosteroid was normal at $189 \mathrm{nmol} / 1(6.8 \mu \mathrm{g} / 100 \mathrm{ml})$, and her overnight urine contained $254 \mathrm{nmol}(92 \mu \mathrm{g})$; on a subsequent occasion a repeat overnight collection gave a raised value of $389 \mathrm{nmol}$ $(141 \mathrm{~kg})$. The diagnosis of bilateral hyperplasia was confirmed at operation. The overnight specimens from all the other patients with Cushing's syndrome contained more than $276 \mathrm{nmol}(100 \mathrm{~kg})$ of 11 -hydroxycorticosteroids, the highest containing $2663 \mathrm{nmol}(965 \mu \mathrm{g})$.

\section{Discussion}

Our results confirm that there is a circadian rhythm in the urinary excretion of adrenal steroids in normal people, the lowest levels occurring during the night. In patients with Cushing's syndrome this rhythm is not so apparent, and the nocturnal excretion is much higher. ${ }^{2}{ }^{3}$ We therefore thought that patients with adrenocortical overactivity might be identified by their higher overnight urinary excretion of 11-hydroxycorticosteroids, and this was found to be so.

Our data were obtained solely from women because only one man with Cushing's syndrome was admitted during the study. The overnight 11-hydroxycorticosteroid level in this man with an adrenal carcinoma was grossly raised at $5382 \mathrm{nmol}(1950 \mu \mathrm{g})$. Thus this test is probably applicable to both men and women.

Clinical suspicion of Cushing's syndrome is often aroused by obesity and hirsutism in women, particularly if it is associated with hypertension or diabetes mellitus, but it is not practicable to investigate all such patients in hospital. This simple screening test, requiring only the collection of an overnight urine specimen for 11-hydroxycorticosteroid estimation, has proved useful in selecting those outpatients who require more extensive investigation. Our data indicate that levels over $276 \mathrm{nmol}(100 \mu \mathrm{g})$ are highly indicative of adrenocortical overactivity and merit further action. Clearly the diagnosis of such a serious disorder cannot be completely excluded by one normal result, but if doubt persists the test can easily be repeated.

We are indebted to Dr Peter Sheridan and Dr John Betts for organising the collection of many urine specimens and to Mrs Elaine Bilton and $\mathrm{Mr}$ Harold Martin for excellent technical help. This work was supported by the Northcott Devon Medical Foundation and the South Western Regional Health Authority.

\section{References}

${ }^{1}$ Mattingly, D, and Tyler, C M, British Medical fournal, 1967, 4, 394

2 Vagnucci, A I, et al, fournal of Clinical Endocrinology and Metabolism, $1965,25,1331$.

${ }^{3}$ Kobberling, J, and von zur Muhlen, A, Fournal of Clinical Endocrinology and Metabolism, 1974, 38, 313.

${ }^{4}$ Mattingly, D, and Tyler, C M, British Medical fournal, 1972, 3, 17.

5 Mattingly, D, et al, Lancet, 1964, 2, 1046

\title{
Enhanced $\mathrm{HBsAb}$ production in pathogenesis of fulminant viral hepatitis type $B$
}

\author{
I L WOOLF, N EL SHEIKH, HELEN CULLENS, W M LEE, A L W F EDDLESTON, \\ ROGER WILLIAMS, A J ZUCKERMAN
}

British Medical fournal, 1976, 2, 669-671

\section{Summary}

The possible importance of humoral immunity in the pathogenesis of fulminant hepatitis was investigated by comparing 17 patients with fulminant hepatitis type $B$ with 20 patients with severe but non-fulminant disease. Hepatitis B surface antigen (HBsAg) was cleared from the serum significantly faster $(P<0.001)$ in those with fulminant hepatitis, and in $41 \%$ anti-HBsAg (HBsAb) was detectable by radioimmunoassay (RIA) at presentation. In all 11 sera from patients with fulminant hepatitis

\footnotetext{
Liver Unit, King's College Hospital and Medical School, London SE5 8RX

I L WOOLF, BM, MRCP, lecturer

N EL SHEIKH, MB, BS, research fellow

HELEN CULLENS, ANZIMLT, senior technician

W M LEE, MD, visiting research fellow (present address: Department of Medicine, Columbia University, College of Physicians and Surgeons, New York)

A L W F EDDLESTON, DM, MRCP, senior lecturer

ROGER WILLIAMS, MD, FRCP, director and consultant physician
}

Hepatitis Research Unit, London School of Hygiene and Tropical Medicine, London WC1

A J ZUCKERMAN, MD, DSC, professor of microbiology that were examined by electron microscopy aggregates of $\mathrm{HBs} A g$ and $\mathrm{HBs} A b$ were seen. In contrast, $\mathrm{HBs} A b$ was never detected by RIA in those with non-fulminant hepatitis, and in only one serum specimen (5\%) were aggregates seen on electron microscopy. A significant sex difference between fulminant and non-fulminant hepatitis was observed, $65 \%$ of patients with fulminant hepatitis but only $15 \%$ of patients with non-fulminant hepatitis being women $(P<0.01)$. An enhanced production of HBsAb in fulminant hepatitis, by leading to free HBsAb in portal blood, may cause an Arthus reaction in the sinusoids of the liver with ensuing ischaemic necrosis of hepatocytes.

\section{Introduction}

The reasons why viral hepatitis may occasionally follow a fulminant course are poorly understood. It may occur more often after type B infections, ${ }^{1}$ and factors such as the dose, virulence, and strain of the virus have been incriminated. ${ }^{2-4}$ It has been suggested that in the presence of a normal cellmediated immune response the outcome of an attack of acute hepatitis depends on the number of infected liver cells. ${ }^{5}$ Almeida and Waterson, ${ }^{6}$ however, in an electron microscopic study, described the presence of large immune complexes characteristic of antibody excess in the serum of one patient who died from fulminant hepatitis and speculated on the patho- 